\title{
Stem cell therapy for inflammatory bowel disease
}

\author{
Kanna Nagaishi · Yoshiaki Arimura • \\ Mineko Fujimiya
}

Received: 5 January 2015/Accepted: 5 January 2015/Published online: 25 January 2015

(C) Springer Japan 2015

\begin{abstract}
Inflammatory bowel disease (IBD) could be curable by "immune rest" and correction of the genetic predisposition inherent in allogeneic hematopoietic stem cell transplantation. However, balancing risks against benefits remains challenging. The application of mesenchymal stem cells (MSCs) serving as a site-regulated "drugstore" is a recent concept, which suggests the possibility of an alternative treatment for many intractable diseases such as IBD. Depending on the required function of MSC, such as a cell provider, immune moderator, and/or trophic resource, MSC therapy should be optimized to maximize its therapeutic benefit. Therapeutic effects do not always require full engraftment of MSCs. Therefore, optimization of pleiotropic gut trophic factors produced by MSCs, which favoring not only regulating immune responses but also promoting tissue repair, must directly enhance new drug discoveries for treatment of IBD. Stem cell biology holds great promise for a new era of cell-based therapy, sparking considerable interest among scientists, clinicians, and patients. However, the translational arm of stem cell science remains in a relatively primitive state.
\end{abstract}

The authors K. Nagaishi and Y. Arimura contributed equally to this work.

K. Nagaishi $(\bowtie) \cdot$ M. Fujimiya

Second Department of Anatomy, Sapporo Medical University,

S-1, W-17, Chuo-ku, Sapporo 060-8556, Japan

e-mail: kanna@sapmed.ac.jp

M. Fujimiya

e-mail: fujimiya@sapmed.ac.jp

Y. Arimura

Department of Gastroenterology, Rheumatology and Clinical Immunology, Sapporo Medical University, Sapporo, Japan

e-mail: arimura0330@gmail.com
Although several clinical studies using MSCs have been initiated, early results suggest several inherent problems. In each study, optimization of MSC therapy appears to be the most urgent problem, and can be resolved only by scientifically unveiling the mechanisms of therapeutic action. In the present review, the authors outline how such information would facilitate the critical steps in the paradigm shift from basic research on stem cell biology to clinical practice of regenerative medicine for conquering IBD in the near future.

Keywords Inflammatory bowel disease - Immunemediated inflammatory disease $\cdot$ Mesenchymal stem cells . Hematopoietic stem cell transplantation $\cdot$ MSC-conditioned medium

\section{Introduction}

Although intestinal wound healing comprises each step of epithelial restitution, proliferation, and maturation, the process is comprehensively divided into four phases: hemostasis, inflammation, proliferation, and remodeling [1]. The pathogenesis of inflammatory bowel disease (IBD) involves multiple complex inter-relationships among genetic predisposition, environmental factors, and immunological abnormalities.

Genome-wide association studies (GWASs) suggest impairments of innate defense based on normal microbial recognition and clearance play a crucial role toward the risk of developing Crohn's disease (CD) [2-4]. Furthermore, pro-inflammatory immune responses are likely important given the association of variants in the interleukin-23 (IL-23) pathway, such as $I L-12 B$ and $I L-23$ receptor $(I L-23 R)$, with IBD. The actions of IL-23 have 
largely been linked to Th17 cells, with the most recently discovered $\mathrm{CD}^{+}{ }^{+} \mathrm{T}$ cell subset thought to contribute to many immune-mediated inflammatory diseases (IMID) [5]. Intriguingly, this indicates the extreme differences in IBD susceptible genes identified by recent GWASs [6] among the populations examined. This is strongly suggested either the true IBD disease susceptibility genes which have not been discovered are hidden by a race difference or the genetic predisposition of Japanese IBD could be quite different from western IBD.

Unfortunately, established therapy for IBD has been dominated by a focus on inflammation in the woundhealing process and on immunological abnormalities in its pathogenesis. The optimal IBD therapy aiming for a complete cure should not only block inflammation but also enhance proliferation and coordinate remodeling during the healing process. Furthermore, when examining the overall depiction of health and disease in the intestine, intestinal homeostasis should be regulated by extra-intestinal as well as local machinery. Consequently, therapeutic targets should transform inflammatory cells to intestinal (stem) cells, stromal cells, or bone marrow (stem) cells, and biologics should evolve into stem cell-based or gene-based therapy. Regenerative medicine translated from stem cell biology must provide a backbone to such a paradigm shift in the next-generation therapeutics of IBD.

Since the identity of bona fide mesenchymal stem cells (MSCs) and their specific contributions to these various beneficial effects have remained enigmatic, cultureexpanded multipotent mesenchymal stromal cells (MSCs) are entirely dealt throughout the text in this review. The latter terms are defined as plastic adherent cells that must express CD105, CD90, and CD73 but lack not only the expression of pan-leukocyte (CD45), endothelial, or primitive hematopoietic (CD34), monocyte (CD14 or CD11b), or B cell (CD79a or CD19) markers, but also the expression of HLA class II (HLA-DR) surface antigen, and show tri-lineage differentiation into osteoblasts, adipocytes, and chondrocytes [7].

\section{HSCT and IBD}

The concept and practice of hematopoietic stem cell transplantation (HSCT) as a primary treatment for IMIDs such as IBD began in the late 1990s [8]. Allogeneic HSCT should theoretically be more preferable as a cure than autologous HSCT, as there is a graft-versus-host reaction that removes the presumed disease-causing $\mathrm{T}$ cells that might survive during conditioning [9]. However, since allogeneic transplantation is associated with higher transplant-related morbidity and mortality owing to graft-versus-host disease (GvHD), autologous transplants have been performed more frequently for IMIDs. The rationale of autologous HSCT for IMIDs is to reset the immune system, that is, to generate new self-tolerant lymphocytes after chemotherapy-induced elimination of self- or auto-reactive lymphocytes (i.e., lymph-ablation). Allogeneic HSCT is based on the rationale of both a reset of the immune system and the correction of the genetic predisposition to disease in the immune system by replacing HSCs harboring causative polymorphisms with non-disease-prone HSCs from a healthy donor.

To date, 26 IBD cases consisting of $21 \mathrm{CD}$ and five UC patients who underwent HSCT (eight autologous and 18 allogeneic) for cancer have been reported [8, 10-18]. Overall, 24 of $27(88.9 \%)$ patients have achieved clinical remission in the follow-up periods of 0.5 to 8 years. All three of the patients who suffered from relapses underwent autologous HSCT. These results suggest that HSCT may result in longlasting remission without any additional medications for IBD in most patients. There have been four transplant-related infectious deaths, which accounted for a $14.8 \%$ mortality rate. Interestingly, of the six patients participating in one of the studies (based in Seattle), the one patient who relapsed 18 months after transplantation had mixed chimeras, whereas the four patients who achieved sustained remission were assumed to have complete bone marrow chimeras [17]. Overall, all of the studies support the notion that "immune reset" even under "lymph-ablation" in the autologous transplantation can induce long-lasting remissions in IBD patients. In contrast, in a separate study, a patient without IBD undergoing allogeneic HSCT developed severe CD soon after transplantation [19]. An investigation showed that the transplanted stem cells harbored pathogenic NOD2 mutations.

The therapeutic efficacy of autologous HSCT has been previously suggested in studies consisting of small numbers of patients and is now being evaluated in controlled trials in which the study sizes were more substantial and follow-up periods were longer [20-31]. The first phase I study [22] included 12 patients with active $\mathrm{CD}$, which were refractory to conventional therapies including anti-TNF $\alpha$ treatment. There was an early and sustained clinical remission in 11 of the patients $(91.7 \%)$ after a median follow-up of 18.5 months. One patient experienced CD relapse at 15 months post-transplantation. A second phase I/II study included four refractory patients [23]. Three of the four $(75.0 \%)$ patients had sustained remission after a median follow-up of 16.5 months. All patients in both series tolerated HSCT well and no mortality was observed.

Observations of additional case reports published to date also suggest that sustained clinical remission with HSCT is initially likely to result from lymph-ablation by drugs used 
in the conditioning regimen; there may be a later effect of altered immune reconstitution [20, 21, 24]. Burt et al. reported the phase I/II study of autologous non-myeloablative (lymph-ablative) HSCT in 24 patients with severe anti-TNF refractory CD [25]. The percentage of clinical relapse-free survival, which was defined as the percentage of patients who were not required to restart $\mathrm{CD}$ medical therapy after transplantation, was $91 \%$ at 1 year, $63 \%$ at 2 years, $57 \%$ at 3 years, $39 \%$ at 4 years, and $19 \%$ at 5 years. Additionally, Clerici et al. enrolled seven patients with active refractory $\mathrm{CD}(\mathrm{CDAI} \geq 250)$ in a pilot phase I/II study of autologous HSCT without CD34+ selection [26]. After 12 months, five out of six patients $(83.3 \%)$ maintained clinical and endoscopic remission without further treatment. Only one relapse was observed, requiring an urgent small bowel major resection. Full responders had higher regulatory T-cells (T-regs) and lower interferon (IFN)- $\gamma$ and IL-12 levels than those of relapsed patients. By contrast, T-regs decreased while IL-12 and TLR2 expression levels increased in the only patient who relapsed. These findings confirm that autologous HSCT may result in both high rates of clinical remission and endoscopic mucosal healing. Moreover, HSCT without CD34+ selection seems to be a safer, easier, and less expensive procedure. Hasselblatt et al. reported on the outcome of 12 patients with refractory CD treated with autologous PBSCT [27]. They showed that seven out of nine patients $(77.8 \%)$ had relapsed during follow-up periods (6 months to 10.3 years), but disease activity could be controlled by low-dose corticosteroids and conventional immunosuppressive therapy. Finally, Hawkey recently published an interim analysis of a multicenter, prospective, randomized phase III study designated as the Autologous Stem Cell Transplantation International Crohn's Disease (ASTIC) trial [30]. Autologous HSCT in CD appeared to offer substantial benefits, albeit in fewer patients was implied in the case reports. Moreover, a particularly important finding from this study was that the mobilization was only the benefit of this therapy. He concluded that because of the high level of serious adverse events in the study, the place of stem-cell transplantation in CD must await final results before definitive evaluation can be made.

Although these observations show the limitations of autologous HSCT while underscoring a curative trend of allogeneic HSCT, which was justified based on the monogenic cause of the disease, the balance between risks and benefits remains more ambiguous in the allogeneic transplantation than the autologous transplantation [32]. To increase the applicability of HSCT for IBD, improvement of safety is a top priority. Currently, a phase I trial assessing toxicity and efficacy of non-myeloablative allogeneic HSCT in CD is being performed (http://www.clin icaltrials.gov/ct2/show/NCT01288053).

\section{MSC and IBD}

MSCs are noted for active proliferation, plastic differentiation, strong immunomodulation, low immunogenicity, and abundant trophic factor production. Collectively, the wide range of advantageous effects of MSCs in vivo, from cell replacement and immunosuppression to trophic actions, extend the ability to use in regenerative medicine and immune intervention, the details of which can be found in the outstanding review of MSCs as a cell provider by Richardson et al. [33]. Successful preclinical studies using MSCs in models of autoimmunity, inflammation, and tissue damage have paved the way for clinical trials. Le Blanc et al. [34] reported the GvHD case of a patient who was successfully treated with an immunosuppressive effect of MSCs in vivo, rather than an induction of tolerance. Since then, in humans, ex vivo-expanded allogeneic MSCs have been infused in several phase I studies [35-37]. At present, a total of 311 clinical trials with MSCs are registered in the Clinical Trials.gov database (status October 27, 2014). No adverse events during or after MSC infusion have been observed to date.

In CD, MSCs have been tested in clinical trials for two indications, namely fistulizing and luminal disease [3846]. In fistulizing disease, the differentiation potential of MSCs is thought to be of crucial importance to achieve fistula tract closure. Encouraging results from studies using locally administered MSCs to treat complex perianal fistulae support the use of this approach for patients unresponsive to infliximab [38-44]. For luminal disease, the rationale for the use of MSCs lies mainly in their immunosuppressive capacity. Several phase I and II trials for luminal CD have been performed as well. A phase I trial, which was performed by Duijvestein et al. [45] using autologous bone marrow-derived MSCs (bMSCs) for luminal CD, showed improvement in three out of ten patients, of which none achieved remission. Another group treated four $\mathrm{CD}$ and three ulcerative colitis patients with allogeneic MSCs. Five patients achieved remission, of which two stayed in remission for over 2 years [46]. Recently, preliminary results of a phase II trial analyzing the use of allogeneic bone marrow-derived MSCs for refractory luminal CD were presented (http://clinicaltrials. gov/ct2/show/NCT01090817). Twelve patients out of 14 were improved, including eight patients with remission and seven patients of the endoscopic improvement [47]. These studies were not designed to assess efficacy, but did demonstrate the prospective efficacy and safety of intravenous (i.v.) infusion of MSCs. This notion is supported by a recent meta-analysis analyzing the safety of i.v. infusion of MSCs for many different indications. MSC infusion is associated with transient fever but not with acute reactions, such as infusion toxicity, organ system complications, infections, malignancies, or death [48]. 
However, there are inconsistent results between each study. Long-term adverse effects and the mechanisms of the therapeutic action remain unproven. Since the transplantation efficacy of MSC is extremely poor and the duration when MSC-derived cells are detectable in the impaired intestinal tissues is short, maximum benefit from their therapeutic actions will require more efficient engraftment, proliferation, and/or differentiation of repopulating MSCs in recipient tissues. The likelihood of achieving this remains uncertain. In our experiments, we found a wide array of cues in the micro-milieu for MSC engraftment in experimental colitis; MSC engrafted into the intestinal epithelial region under certain conditions [49] and into stromal tissues under other conditions [50]. In either case, engrafted MSCs were so scarce that we could not observe their dynamics in the recipient intestine. Previous studies also present conflicting reports on the role of MSCs on carcinogenesis [51, 52]. Surprisingly, MSCs can promote or inhibit carcinogenesis depending on the micromilieu cue in our own study (data not shown).

Stem cell-based therapy with curative intent is growing in importance as the next-generation therapy for IBD. However, it is essential to set clear objectives regarding their therapeutic targets and to understand the precise mechanism of repair in each clinical setting to ensure optimal MSC therapy. All issues, such as source and types of MSC (autologous or allogeneic), the quality control of prepared MSCs (homogenous/monoclonal or heterogeneous/polyclonal), procedure of administration (route, schedule, dose, pretreatment with cytokines or chemokines, etc.) and factors that raise transplantation efficacy and control appropriate differentiation in the desired location, must be understood clearly. Even now, the process and evaluation criteria to choose autologous or allogeneic MSCs remains poorly understood. Allogeneic MSCs are regarded as a preferred source as they would allow treatment with a ready-to-use, off-the-shelf product, available for a large number of patients. Since exposure of inflammatory mediators up-regulates HLA class I and induces class II molecules, MSCs may be immune privilege cells. However, the absence of classic co-stimulatory molecules would lead to anergy of $\mathrm{T}$ lymphocytes. In addition, there is evidence of immune recognition of allogeneic MSCs by the host immune system, which may lead clearance of the cells. However, some evidence suggests that those alloresponses can be readily controlled by the inherent immune-modulatory properties of MSCs [53]. Autologous MSCs, on the contrary, would be favorable because they do not suffer from immune rejection. However, it is not clear whether autologous MSCs which are expanded from patients with inflammatory or autoimmune diseases may be fully functional [54]. Surprisingly, the therapeutic effects of MSCs do not depend on their full engraftment, but rely on the capacity of MSCs to inhibit pathogenic immune responses and to release trophic factors favoring tissue repair [55-57]. We found that the conditioned medium of MSCs including pleiotropic gut trophic factors promoted intestinal epithelial repair [58]. Consequently, we believe that discovery of the strategy to optimize MSC and ingredient analysis of MSC-conditioned medium (MSC$\mathrm{CM}$ ) would open new avenues for drug discovery and establish a basis for cell-based therapy for IBD (Fig. 1).

\section{Intestinal stem cells and other stem cells in IBD}

Recently, gastrointestinal stem cell culture has been established in humans as well as rodents [59-62]; transplantation of these stem cells opens real possibilities of novel approaches to intractable gastrointestinal diseases such as IBD. However, transplantation of intestinal stem cells remains in its infancy, and readers should refer elsewhere for more information on this subject. Several other stem cells have been explored in preclinical settings as well. Induced pluripotent stem cells (iPSCs) are pluripotent cells derived from terminally differentiated cells by dedifferentiation. The generation of iPSCs creates the possibility of generating tissue-specific cells, including HSCs [63-68] or MSCs [69-76], but further study is required before clinical application of iPSCs can commence.

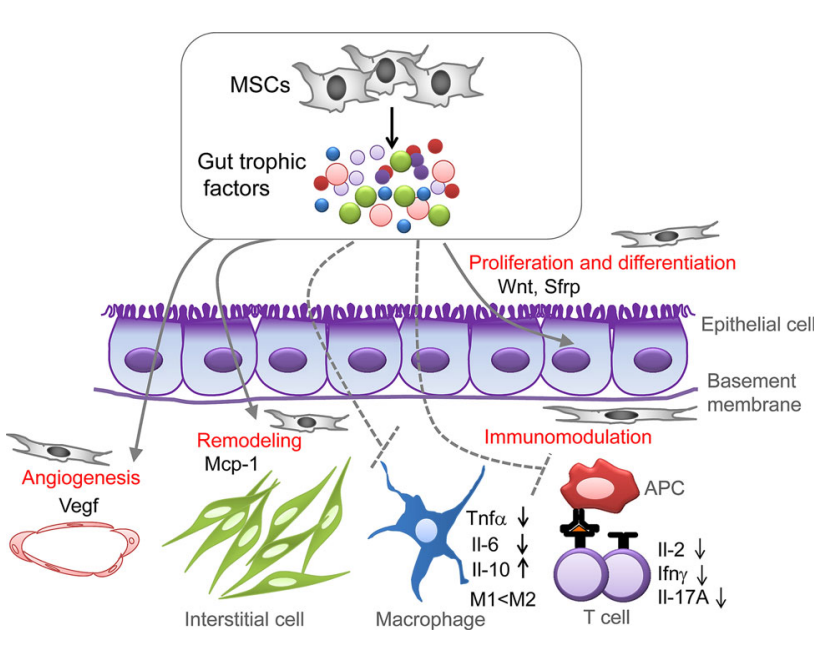

Fig. 1 Concept of the intestinal mucosal regeneration by gut trophic factors derived from MSC. MSC-CM contains pleiotropic gut trophic factors that promote intestinal epithelial cell proliferation and differentiation, immunomodulation, interstitial remodeling, and angiogenesis on each component of the mucosal healing machinery. Individual cytokines and growth factors in the MSC-CM are listed in the figure. To determine the ideal preconditions for MSCs to enhance the production of beneficial gut trophic factors and to optimize MSC$\mathrm{CM}$ as a chemical compound will lead to higher and broader therapeutic potential of MSC therapy and innovative drug discovery for IBD 
Table 1 Requirements for complete cure of IBD and ability for radical cure of various stem cell treatments

\begin{tabular}{|c|c|c|c|c|c|c|}
\hline & \multicolumn{2}{|l|}{ HSCT } & \multicolumn{2}{|l|}{ MSC treat } & \multicolumn{2}{|c|}{ Intestinal stem cell transplantation } \\
\hline & Allogenic & Autologous & Allogenic & Autologous & Allogenic & Autologous \\
\hline \multicolumn{7}{|l|}{ Immune reconstruction } \\
\hline Pre-treatment for bone marrow cells & (?) & (?) & $\times$ & $\times$ & $\bigcirc$ & $\times$ \\
\hline Immunoregulatory action & $\times$ & $\times$ & $\bigcirc$ & $\bigcirc$ & $x$ & $\times$ \\
\hline \multicolumn{7}{|l|}{ Modification of genetic predisposition } \\
\hline \multicolumn{7}{|l|}{ Bone marrow cell } \\
\hline HSC (immunocompetent cell) & (?) & $\times$ & $\times$ & $x$ & $x$ & $\times$ \\
\hline Niche cell & $x$ & $x$ & $\bigcirc$ & $\times$ & $x$ & $x$ \\
\hline Intestinal epithelial cell & $\times$ & $\times$ & $\times$ & $\times$ & ( ) & $x$ \\
\hline \multicolumn{7}{|l|}{ Mucosal healing } \\
\hline Epithelial restitution & $\mathrm{u} / \mathrm{n}$ & $\mathrm{u} / \mathrm{n}$ & $\bigcirc$ & $\bigcirc$ & $\bigcirc$ & $\bigcirc$ \\
\hline Anti-inflammatory & $\mathrm{u} / \mathrm{n}$ & $\mathrm{u} / \mathrm{n}$ & $\bigcirc$ & $\bigcirc$ & $x$ & $\times$ \\
\hline Proliferation & $\mathrm{u} / \mathrm{n}$ & $\mathrm{u} / \mathrm{n}$ & $\bigcirc$ & $\bigcirc$ & $\bigcirc$ & $\bigcirc$ \\
\hline Differentiation/maturation & $\mathrm{u} / \mathrm{n}$ & $\mathrm{u} / \mathrm{n}$ & $\bigcirc$ & $\bigcirc$ & $\bigcirc$ & $\bigcirc$ \\
\hline
\end{tabular}

$H S C T$ hematopoietic stem cell transplantation, MSC mesenchymal stem cell, $H S C$ hematopoietic stem cell, $u / n$ unknown

Taken together, immune reconstruction and modification of genetic predisposition of hematopoietic stem cells are important for radical cure of IBD. As a summary of the arguments of these various transplant treatments, the requirements to achieve a complete cure of IBD are listed in Table 1. The double circle shows the therapeutic approach that completely suppresses each pathologic factor, the single circle shows that of partial suppression, and a cross indicates that it does not meet the condition.

\section{Future perspectives}

Notwithstanding the enticing perspective of immune reset, it is unrealistic to believe that autologous HSCT can eradicate immune diseases because a genetic predisposition may remain unmodified in the transplanted stem cells and disease recurrence remains a potential risk. The last decade has seen a remarkable shift in our appreciation of the potential uses for MSCs instead of HSCs. Now the challenge is the scientific evaluation of MSC therapy against conventional treatments for IBD in randomized controlled trials. The possibility of using allogeneic MSCs and immunomodulatory properties of MSCs in preventing GvHD is a promising and safer perspective for testing this treatment in IBD. Furthermore, optimization of pleiotropic gut trophic factor produced by MSCs should potentiate new drug discoveries for IBD. Significant hurdles remain regarding the design and interpretation of studies on MSCs, including patient selection, disease stage, disease activity, and long-term safety.
Acknowledgments We are very grateful to Dr. Gregory Wheeler, Assistant Professor in the Department of Liberal Arts and Science, Sapporo Medical University for linguistic assistance.

Conflict of interest The authors declare that they have no conflict of interest.

\section{References}

1. Stadelmann WK, Digenis AG, Tobin GR. Physiology and healing dynamics of chronic cutaneous wounds. Am J Surg. 1998; 176:26S-38S.

2. Jostins L, Ripke S, Weersma RK, et al. Host-microbe interactions have shaped the genetic architecture of inflammatory bowel disease. Nature. 2012;491:119-24.

3. Cho JH, Brant SR. Recent insights into the genetics of inflammatory bowel disease. Gastroenterology. 2011;140:1704-12.

4. Raelson JV, Little RD, Ruether A, et al. Genome-wide association study for Crohn's disease in the Quebec founder population identifies multiple validated disease loci. Proc Natl Acad Sci USA. 2007;104:14747-52.

5. Steinman L. A brief history of $\mathrm{T}(\mathrm{H}) 17$, the first major revision in the $\mathrm{T}(\mathrm{H}) 1 / \mathrm{T}(\mathrm{H}) 2$ hypothesis of $\mathrm{T}$ cell-mediated tissue damage. Nat Med. 2007;13:139-45.

6. Van Limbergen J, Wilson DC, Satsangi J. The genetics of Crohn's disease. Annu Rev Genomics Hum Genet. 2009;10:89-116.

7. Lv FJ, Tuan RS, Cheung KM, et al. Concise review: the surface markers and identity of human mesenchymal stem cells. Stem Cells. 2014;32:1408-19.

8. Drakos PE, Nagler A, Or R. Case of Crohn's disease in bone marrow transplantation. Am J Hematol. 1993;43:157-8.

9. Marmont AM. Immunoablation followed or not by hematopoietic stem cells as an intense therapy for severe autoimmune diseases. New perspectives, new problems. Haematologica. 2001;86:337-45.

10. Ditschkowski M, Einsele H, Schwerdtfeger R, et al. Improvement of inflammatory bowel disease after allogeneic stem-cell transplantation. Transplantation. 2003;75:1745-7. 
11. Talbot DC, Montes A, Teh WL, et al. Remission of Crohn's disease following allogeneic bone marrow transplant for acute leukaemia. Hosp Med. 1998;59:580-1.

12. Kashyap A, Forman SJ. Autologous bone marrow transplantation for non-Hodgkin's lymphoma resulting in long-term remission of coincidental Crohn's disease. Br J Haematol. 1998;103:651-2.

13. Musso M, Porretto F, Crescimanno A, et al. Crohn's disease complicated by relapsed extranodal Hodgkin's lymphoma: prolonged complete remission after unmanipulated PBPC autotransplant. Bone Marrow Transplant. 2000;26:921-3.

14. Soderholm JD, Malm C, Juliusson G, et al. Long-term endoscopic remission of Crohn disease after autologous stem cell transplantation for acute myeloid leukaemia. Scand J Gastroenterol. 2002;37:613-6.

15. Anumakonda V, Hayee B, Chung-Faye G. Remission and relapse of Crohn's disease following autologous haematopoietic stem cell transplantation for non-Hodgkin's lymphoma. Gut. 2007;56:1325.

16. Marti JL, Mayordomo JI, Isla MD, et al. PBSC autotransplant for inflammatory bowel disease (IBD): a case of ulcerative colitis. Bone Marrow Transplant. 2001;28:109-10.

17. Lopez-Cubero SO, Sullivan KM, McDonald GB. Course of Crohn's disease after allogeneic marrow transplantation. Gastroenterology. 1998;114:433-40.

18. Hawkey CJ. Stem cell transplantation for Crohn's disease. Best Pract Res Clin Haematol. 2004;17:317-25.

19. Sonwalkar SA, James RM, Ahmad T, et al. Fulminant Crohn's colitis after allogeneic stem cell transplantation. Gut. 2003;52:1518-21.

20. Kreisel W, Potthoff K, Bertz H, et al. Complete remission of Crohn's disease after high-dose cyclophosphamide and autologous stem cell transplantation. Bone Marrow Transplant. 2003;32:337-40.

21. Scime R, Cavallaro AM, Tringali $S$, et al. Complete clinical remission after high-dose immune suppression and autologous hematopoietic stem cell transplantation in severe Crohn's disease refractory to immunosuppressive and immunomodulator therapy. Inflamm Bowel Dis. 2004;10:892-4.

22. Oyama Y, Craig RM, Traynor AE, et al. Autologous hematopoietic stem cell transplantation in patients with refractory Crohn's disease. Gastroenterology. 2005;128:552-63.

23. Cassinotti A, Annaloro C, Ardizzone S, et al. Autologous haematopoietic stem cell transplantation without CD34+ cell selection in refractory Crohn's disease. Gut. 2008;57:211-7.

24. Glocker EO, Kotlarz D, Boztug K, et al. Inflammatory bowel disease and mutations affecting the interleukin-10 receptor. N Engl J Med. 2009;361:2033-45.

25. Burt RK, Craig RM, Milanetti F, et al. Autologous nonmyeloablative hematopoietic stem cell transplantation in patients with severe anti-TNF refractory Crohn disease: long-term followup. Blood. 2010;116:6123-32.

26. Clerici M, Cassinotti A, Onida F, et al. Immunomodulatory effects of unselected haematopoietic stem cells autotransplantation in refractory Crohn's disease. Dig Liver Dis. 2011;43:946-52.

27. Hasselblatt P, Drognitz K, Potthoff K, et al. Remission of refractory Crohn's disease by high-dose cyclophosphamide and autologous peripheral blood stem cell transplantation. Aliment Pharmacol Ther. 2012;36:725-35.

28. Kountouras J, Sakellari I, Tsarouchas G, et al. Autologous haematopoietic stem cell transplantation in a patient with refractory Crohn's disease. J Crohns Colitis. 2011;5:275-6.

29. Hommes DW, Duijvestein M, Zelinkova Z, et al. Long-term follow-up of autologous hematopoietic stem cell transplantation for severe refractory Crohn's disease. J Crohns Colitis. 2011;5:543-9.
30. Hawkey CJ. Stem cells as treatment in inflammatory bowel disease. Dig Dis. 2012;30(Suppl 3):134-9.

31. Burt RK, Traynor A, Oyama Y, Craig R. High-dose immune suppression and autologous hematopoietic stem cell transplantation in refractory Crohn disease. Blood. 2003;101:2064-6.

32. Bohgaki T, Atsumi T, Koike T. Multiple autoimmune diseases after autologous stem-cell transplantation. $\mathrm{N}$ Engl J Med. 2007;357:2734-6.

33. Richardson SM, Hoyland JA, Mobasheri R, et al. Mesenchymal stem cells in regenerative medicine: opportunities and challenges for articular cartilage and intervertebral disc tissue engineering. J Cell Physiol. 2010;222:23-32.

34. Le Blanc K, Rasmusson I, Sundberg B, et al. Treatment of severe acute graft-versus-host disease with third party haploidentical mesenchymal stem cells. Lancet. 2004;363:1439-41.

35. Koc ON, Day J, Nieder M, et al. Allogeneic mesenchymal stem cell infusion for treatment of metachromatic leukodystrophy (MLD) and Hurler syndrome (MPS-IH). Bone Marrow Transplant. 2002;30:215-22.

36. Horwitz EM, Gordon PL, Koo WK, et al. Isolated allogeneic bone marrow-derived mesenchymal cells engraft and stimulate growth in children with osteogenesis imperfecta: implications for cell therapy of bone. Proc Natl Acad Sci USA. 2002;99:8932-7.

37. Le Blanc K, Frassoni F, Ball L, et al. Mesenchymal stem cells for treatment of steroid-resistant, severe, acute graft-versus-host disease: a phase II study. Lancet. 2008;371:1579-86.

38. Garcia-Olmo D, Garcia-Arranz M, Herreros D, et al. A phase I clinical trial of the treatment of Crohn's fistula by adipose mesenchymal stem cell transplantation. Dis Colon Rectum. 2005;48:1416-23.

39. Garcia-Olmo D, Herreros D, Pascual I, et al. Expanded adiposederived stem cells for the treatment of complex perianal fistula: a phase II clinical trial. Dis Colon Rectum. 2009;52:79-86.

40. Ciccocioppo R, Bernardo ME, Sgarella A, et al. Autologous bone marrow-derived mesenchymal stromal cells in the treatment of fistulising Crohn's disease. Gut. 2011;60:788-98.

41. Guadalajara H, Herreros D, De-La-Quintana P, et al. Long-term follow-up of patients undergoing adipose-derived adult stem cell administration to treat complex perianal fistulas. Int $\mathrm{J}$ Colorectal Dis. 2012;27:595-600.

42. Herreros MD, Garcia-Arranz M, Guadalajara H, et al. Autologous expanded adipose-derived stem cells for the treatment of complex cryptoglandular perianal fistulas: a phase III randomized clinical trial (FATT 1: fistula Advanced Therapy Trial 1) and long-term evaluation. Dis Colon Rectum. 2012;55:762-72.

43. de la Portilla F, Alba F, Garcia-Olmo D, et al. Expanded allogeneic adipose-derived stem cells (eASCs) for the treatment of complex perianal fistula in Crohn's disease: results from a multicenter phase I/IIa clinical trial. Int $\mathrm{J}$ Colorectal Dis. 2013;28:313-23.

44. Lee WY, Park KJ, Cho YB, et al. Autologous adipose tissuederived stem cells treatment demonstrated favorable and sustainable therapeutic effect for Crohn's fistula. Stem Cells. 2013;31:2575-81.

45. Duijvestein M, Vos AC, Roelofs $\mathrm{H}$, et al. Autologous bone marrow-derived mesenchymal stromal cell treatment for refractory luminal Crohn's disease: results of a phase I study. Gut. 2010;59:1662-9.

46. Liang J, Zhang H, Wang D, et al. Allogeneic mesenchymal stem cell transplantation in seven patients with refractory inflammatory bowel disease. Gut. 2012;61:468-9.

47. Forbes GM, Sturm MJ, Leong RW, et al. A phase 2 study of allogeneic mesenchymal stromal cells for luminal Crohn's disease refractory to biologic therapy. Clin Gastroenterol Hepatol. 2014;12:64-71. 
48. Lalu MM, McIntyre L, Pugliese C, et al. Safety of cell therapy with mesenchymal stromal cells (SafeCell): a systematic review and meta-analysis of clinical trials. PLoS One. 2012;7:e47559.

49. Yabana T, Arimura Y, Tanaka H, et al. Enhancing epithelial engraftment of rat mesenchymal stem cells restores epithelial barrier integrity. J Pathol. 2009;218:350-9.

50. Tanaka H, Arimura Y, Yabana T, et al. Myogenic lineage differentiated mesenchymal stem cells enhance recovery from dextran sulfate sodium-induced colitis in the rat. J Gastroenterol. 2011;46:143-52.

51. Nasuno M, Arimura Y, Nagaishi K, et al. Mesenchymal stem cells cancel azoxymethane-induced tumor initiation. Stem Cells. 2014;3:13-25.

52. Khakoo AY, Pati S, Anderson SA, et al. Human mesenchymal stem cells exert potent antitumorigenic effects in a model of Kaposi's sarcoma. J Exp Med. 2006;203:1235-47.

53. Griffin MD, Ritter T, Mahon BP. Immunological aspects of allogeneic mesenchymal stem cell therapies. Hum Gene Ther. 2010;21:1641-55.

54. Dalle Carbonare L, Valenti MT, Zanatta M, et al. Circulating mesenchymal stem cells with abnormal osteogenic differentiation in patients with osteoporosis. Arthritis Rheum. 2009;60:3356-3365.

55. Karnoub AE, Dash AB, Vo AP, et al. Mesenchymal stem cells within tumour stroma promote breast cancer metastasis. Nature. 2007;449:557-63.

56. Uccelli A, Laroni A, Freedman MS. Mesenchymal stem cells for the treatment of multiple sclerosis and other neurological diseases. Lancet Neurol. 2011;10:649-56.

57. Hansson EM, Lindsay ME, Chien KR. Regeneration next: toward heart stem cell therapeutics. Cell Stem Cell. 2009;5:364-77.

58. Watanabe S, Arimura Y, Nagaishi K, et al. Conditioned mesenchymal stem cells produce pleiotropic gut trophic factors. J Gastroenterol. 2014;49:270-82.

59. Jung P, Sato T, Merlos-Suarez A, et al. Isolation and in vitro expansion of human colonic stem cells. Nat Mebd. 2011;17:1225-7.

60. Sato T, Stange DE, Ferrante M, et al. Long-term expansion of epithelial organoids from human colon, adenoma, adenocarcinoma, and Barrett's epithelium. Gastroenterology. 2011;141:1762-72.

61. Yui S, Nakamura T, Sato T, et al. Functional engraftment of colon epithelium expanded in vitro from a single adult Lgr5(+) stem cell. Nat Med. 2012;18:618-23.

62. Watanabe M. Adult tissue stem cell therapy for gastrointestinal diseases. J Gastroenterol Hepatol. 2014;. doi:10.1111/jgh.12555.
63. Grigoriadis AE, Kennedy M, Bozec A, et al. Directed differentiation of hematopoietic precursors and functional osteoclasts from human ES and iPS cells. Blood. 2010;115:2769-76.

64. Inoue-Yokoo T, Tani K, Sugiyama D. Mesodermal and hematopoietic differentiation from ES and iPS cells. Stem Cell Rev. 2013;9:422-34.

65. Kim EM, Manzar G, Zavazava N. Human iPS cell-derived hematopoietic progenitor cells induce T-cell anergy in in vitrogenerated alloreactive $\mathrm{CD} 8(+) \quad \mathrm{T}$ cells. Blood. 2013;121:5167-75.

66. Klump H, Teichweyde N, Meyer C, et al. Development of patient-specific hematopoietic stem and progenitor cell grafts from pluripotent stem cells, in vitro. Curr Mol Med. 2013;13:815-20.

67. Lengerke C, Grauer M, Niebuhr NI, et al. Hematopoietic development from human induced pluripotent stem cells. Ann N Y Acad Sci. 2009;1176:219-27.

68. Mazurier C, Douay L, Lapillonne H. Red blood cells from induced pluripotent stem cells: hurdles and developments. Curr Opin Hematol. 2011;18:249-53.

69. Wu SM, Hochedlinger K. Harnessing the potential of induced pluripotent stem cells for regenerative medicine. Nat Cell Biol. 2011;13:497-505.

70. Chen YS, Pelekanos RA, Ellis RL, et al. Small molecule mesengenic induction of human induced pluripotent stem cells to generate mesenchymal stem/stromal cells. Stem Cells Transl Med. 2012;1:83-95.

71. Eberle I, Moslem M, Henschler R, et al. Engineered MSCs from patient-specific iPS cells. Adv Biochem Eng Biotechnol. 2013;130:1-17.

72. Giuliani M, Oudrhiri N, Noman ZM, et al. Human mesenchymal stem cells derived from induced pluripotent stem cells downregulate NK-cell cytolytic machinery. Blood. 2011;118:3254-62.

73. Liu Y, Goldberg AJ, Dennis JE, et al. One-step derivation of mesenchymal stem cell (MSC)-like cells from human pluripotent stem cells on a fibrillar collagen coating. PLoS One. 2012; 7:e33225.

74. Otsu K, Kishigami R, Oikawa-Sasaki A, et al. Differentiation of induced pluripotent stem cells into dental mesenchymal cells. Stem Cells Dev. 2012;21:1156-64.

75. Teramura $\mathrm{T}$, Onodera $\mathrm{Y}$, Mihara $\mathrm{T}$, et al. Induction of mesenchymal progenitor cells with chondrogenic property from mouseinduced pluripotent stem cells. Cell Reprogram. 2010;12:249-61.

76. Villa-Diaz LG, Brown SE, Liu Y, et al. Derivation of mesenchymal stem cells from human induced pluripotent stem cells cultured on synthetic substrates. Stem Cells. 2012;30:1174-81. 